\title{
Stern-Gerlach Dynamics with Quantum Propagators
}

Bailey C. Hsu

Manuel Berrondo

Jean F. Van Huele

vanhuele@byu.edu

Follow this and additional works at: https://scholarsarchive.byu.edu/facpub

Part of the Quantum Physics Commons

\section{Original Publication Citation}

Hsu, B. C., Berrondo, M., \& Van Huele, J. F. S. (2011). Stern-Gerlach dynamics with quantum propagators. Physical Review A, 83(1), 012109.

\section{BYU ScholarsArchive Citation}

Hsu, Bailey C.; Berrondo, Manuel; and Van Huele, Jean F., "Stern-Gerlach Dynamics with Quantum Propagators" (2011). Faculty Publications. 1852.

https://scholarsarchive.byu.edu/facpub/1852 


\title{
Stern-Gerlach dynamics with quantum propagators
}

\author{
Bailey C. Hsu, ${ }^{*}$ Manuel Berrondo, ${ }^{\dagger}$ and Jean-François S. Van Huele ${ }^{\ddagger}$ \\ Department of Physics and Astronomy, Brigham Young University, Provo, Utah 84602, USA
}

(Received 8 June 2010; published 25 January 2011)

\begin{abstract}
We study the quantum dynamics of a nonrelativistic neutral particle with spin in inhomogeneous external magnetic fields. We first consider fields with one-dimensional inhomogeneities, both unphysical and physical, and construct the corresponding analytic propagators. We then consider fields with two-dimensional inhomogeneities and develop an appropriate numerical propagation method. We propagate initial states exhibiting different degrees of space localization and various initial spin configurations, including both pure and mixed spin states. We study the evolution of their spin densities and identify characteristic features of spin density dynamics, such as the spatial separation of spin components, and spin localization or accumulation. We compare our approach and our results with the coverage of the Stern-Gerlach effect in the literature, and we focus on nonstandard Stern-Gerlach outcomes, such as radial separation, spin focusing, spin oscillation, and spin flipping.
\end{abstract}

DOI: 10.1103/PhysRevA.83.012109

PACS number(s): 03.65.Sq, 03.65.Ta

\section{INTRODUCTION}

The deflection of a beam of silver atoms, achieved by Otto Stern and Walther Gerlach in Frankfurt am Main in 1921-1922, represents a milestone in the development of modern physics [1]. The significance of the Stern-Gerlach effect (SGE) can be argued from many angles: (i) it represented the beginning of a very rich line of atomic beam research [2], (ii) it demonstrated the concept of space quantization [3], (iii) it was later recognized as confirming the existence of spin [4], (iv) it is an early manifestation of nonclassical correlations or entanglement [5], and (v) it has been used as a natural laboratory for the measurement problem [6]. For all these reasons it is an exemplary quantum effect, and many textbooks start from the SGE to develop quantum formalism and quantum intuition [7,8]. The experimental reality of the SGE constrains its theoretical description. As reviewed below, a considerable amount of effort has been devoted to recovering the outcome of Stern-Gerlach experiments within different theoretical frameworks and applying a variety of approximations. Since exact analytic results for the specific conditions of a Stern-Gerlach experiment are difficult to obtain, the main emphasis of the work since 1922 has been to justify the approximations, either by showing their internal consistency or by experimentally corroborating the results they lead to. The justification for the approximations is often missing in the pedagogical literature, where Stern-Gerlach outcomes have increasingly been used as a tool and a test for quantum understanding [9]. This is not very surprising given the significance of SGE in quantum theory, but it highlights the need to clearly delineate the approximations. More recently, the possibility of probing Stern-Gerlach experiments beyond the region of validity of the approximations in order to uncover new (nonstandard) results and understand their quantum significance has been considered $[10,11]$.

\footnotetext{
*bailey.c.hsu@gmail.com

†berrondo@byu.edu

${ }^{\ddagger}$ vanhuele@byu.edu
}

The current work studies both standard and nonstandard Stern-Gerlach dynamics. We are interested in not only treating the SGE fully quantum mechanically to recover the standard result but also extending the study in regions where the standard approximation breaks down. The Stern-Gerlach experiment predates quantum mechanics, and its theoretical description was originally framed in semiclassical terms [12-14], where a differentiated force on the atomic magnet results in its deflection and the quantization of the magnetic moment along the direction of the field inhomogeneity leads to a discrete set of selected trajectories. Semiclassical results are recovered when the expectation values of quantum-mechanical operators are evaluated. Purely quantum-mechanical treatments have been given starting with Bohm [15], who found that the two spin components acquire a momentum kick in opposite directions. This treatment is covered in some quantummechanics texts $[16,17]$. One of the limitations mentioned above pertains to the inhomogeneity of the magnetic field. As we discuss in more depth below, Maxwell's equation $\boldsymbol{\nabla} \cdot \mathbf{B}=\mathbf{0}$ prevents the field and its inhomogeneity to point in the same direction, but implies the existence of a component of the inhomogeneity in a perpendicular direction. This perpendicular inhomogeneity is usually neglected because the expectation value of the perpendicular magnetic moment averages to zero as this magnetic moment precesses around the stronger homogeneous magnetic field. This has been discussed within a classical and semiclassical framework [18]. In our discussion we consider three cases: the standard case where this second (perpendicular) inhomogeneity can be neglected, an alternate field where the inhomogeneity is purely perpendicular to the field, and the case where the second inhomogeneity cannot and should not be neglected. This last case leads to a deviation from the standard result, which could be characterized by the statement "spin-up goes up and spin-down goes down." Another limitation that we address is the localization of the wave packet. Some quantum-mechanical treatments show that plane waves pick up a vertical component of the momentum, which depends on the spin state. Using localized wave packets instead of plane waves allows us to do several things: to represent a beam of finite extent, to study the dependence of the effects on the width of the 
beam and on the location of the beam within the field, and thus to characterize the type of Stern-Gerlach behavior by comparing the width and the position of the beam in the field. We also choose to consider another parameter in this study, namely, the polarization of the beam. We contrast the results for fully coherent (pure) spin states that are usually found in the literature to the totally unpolarized beams. We find that spincoherent states that break the combined geometrical symmetry of the field and the beam can lead to oscillations along the direction of symmetry breaking. We note here that the original Stern-Gerlach experiment was performed with an unpolarized beam of finite cross section. Localization and polarization are actually not independent, for we find that coherent states are typically more favorable to the presence of nonstandard effects. Variations of the quantum-mechanical approach to SGE have been proposed, such as a Bohmian theory of SGE [19] and space-time trajectories of statistical ensembles [20]. The SGE for charged particles has been considered in quantum mechanics [21] or within the Wentzel-KramersBrillouin (WKB) approximation for inhomogeneities along the beam axis (longitudinal SGE) [22]. The $U$-matrix method, originally developed by Kennard [23], was used [24] to develop propagators in inhomogeneous "unphysical" magnetic fields (by this we mean an inhomogeneous magnetic field that does not obey Maxwell's equation $\boldsymbol{\nabla} \cdot \mathbf{B}=0$ ). Scully, Lamb, and Barut revisited the issue for a "physical" SGE [25]. They introduce a quantum-mechanical representation of the time evolution operator, both in coordinate and spin space, a spin propagator. The results [24] and [25] are incomplete, however, as the propagators are not exact and no propagated solutions are given. It should also be pointed out that the historical Stern-Gerlach experiment used a beam of unpolarized silver atoms. However, the typical quantum-mechanical treatment discusses pure states only, usually based on momentum eigenstates $[15,16]$. As we show in this work, the choice matters, for pure and mixed states lead to different features in the SGE. In some quantum-mechanical treatments, nonstandard features have been identified [10,26]. Although some of the results presented below depart from the standard SGE, the specifics are different, and the study of their dependence on the choice of parameters allows us to control their presence to some extent. In particular we find some features that are reminiscent of spin-orbit coupling dynamics [27].

The method that we use to derive both standard and nonstandard results is based on the concept of propagation in time. The propagator $K\left(x, x_{0} ; t, t^{\prime}\right)$ gives the conditional transition amplitude between two position eigenstate vectors $|x\rangle$ and $\left|x_{0}\right\rangle$ over a time interval $t-t^{\prime}$ such that $K\left(x, x_{0} ; t, t^{\prime}\right)=\left\langle x\left|U\left(t, t^{\prime}\right)\right| x_{0}\right\rangle$, where $U\left(t, t^{\prime}\right)$ is the time-evolution operator [28]. For the time-independent problems, $K\left(x, x_{0} ; t, t^{\prime}\right)=K\left(x, x_{0} ; t-t^{\prime}\right)$. Without loss of generality we set $t^{\prime}=0$ in this paper. We generalize the quantum-mechanical propagator approach to spin-dependent Hamiltonians applicable to SG configurations. Because the Hamiltonians involve spin operators, the propagators have a $2 \times 2$ matrix representation for spin-1/2 particles. Several methods for constructing propagators are available in the literature [23,28-34] but none of them addresses the spin-dependent terms in the potential energy. Our goal is to extend some of these methods to spin-dependent potentials of the SG type, that is, potentials combining spin under the form of Pauli matrices and coordinates through magnetic fields that are linear in the coordinates. Some spin-orbit potentials combining spin and momentum have become very important in the area of spintronics [35-37] and analytic spin propagators have been obtained in this case also [27]. Once we have constructed the propagators, we apply them to localized Gaussian wave packets and follow the evolution of the spin dynamics. This Gaussian wave packet treatment goes beyond the delta distribution approach used in [24]. Unlike [24] and [25] we obtain spin separation. As the complexity of the noncommuting operators increases, we replace the analytic expressions with step-by-step numerical propagation, as explained below. This method can be used to verify the analytic results as well as to extend the study to nonstandard results.

This paper is organized as follows. In Sec. II we introduce both analytic and numerical methods to propagate wave packets in systems involving magnetic fields with one-dimensional (1D) and two-dimensional (2D) inhomogeneities. We give the exact analytic quantum propagators for two different 1Dinhomogeneity cases. We also present a numerical propagation method based on the Trotter's formula for exponentials of noncommutative operators for 2D-inhomogeneity cases [38]. In Sec. III we first construct the spin densities in 1D-inhomogeneity systems by applying the specific analytic propagator to an initial Gaussian wave packet in space and polarized along specific directions, and we identify three characteristic features of spin dynamics. We then use a numerical propagation method to generate the wave packet evolution in 2D-inhomogeneity systems and highlight three features of the spin densities. In all cases, we display these features with different values of parameters, namely, interaction strengths and wave packet widths, to demonstrate dependence of the effects on these parameters. We also show to what extent the results differ between initial pure (coherent) states and mixed states. In Sec. IV, we discuss our results and speculate on the consequences.

\section{PROPAGATOR CONSTRUCTION}

\section{A. 1D-inhomogeneity propagation}

We focus on the propagation in the $x z$ plane, which is perpendicular to the beam, in order to study the SG deflection. In this work we follow the convention of the traditional Stern-Gerlach theoretical setup where the $\hat{y}$ axis defines the beam direction and the dimension where no inhomogeneity of the magnetic field is present. Our system is thus explicitly independent of the coordinate $y$ and we can follow the system in successive parallel planes. We define a time $\tau$ that refers to the length of time that the wave packet stays inside the field configurations $\mathbf{B}(x, z)$ of the SG magnet. Therefore, the magnetic field $\mathbf{B}(t)$ experienced by a wave packet for all space can be described by

$$
\mathbf{B}(t)=\left\{\begin{array}{lc}
0, & t<0 \\
\mathbf{B}(x, z), & 0<t<\tau \\
0, & t>\tau .
\end{array}\right.
$$



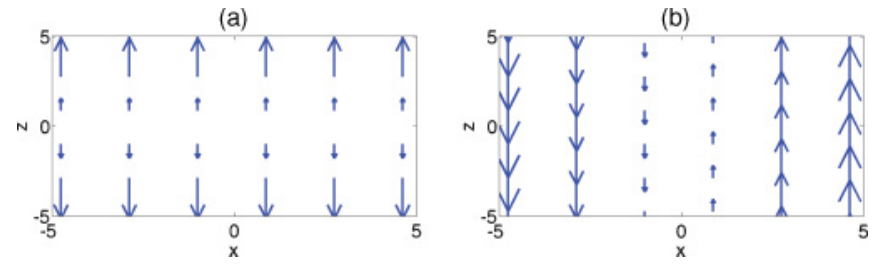

FIG. 1. (Color online) Plots of the magnetic field $\mathbf{B}$. (a) $\mathbf{B}_{1 \mathrm{D}}^{u}=$ $\left(0,0, B_{1} z\right),(\mathrm{b}) \mathbf{B}_{1 \mathrm{D}}^{p}=\left(0,0, B_{1} x\right)$.

In what follows we provide both analytical and numerical constructions of propagators for fields involving $1 \mathrm{D}$ and 2D inhomogeneities, respectively.

In the $1 \mathrm{D}$-inhomogeneity case, we consider two different fields: the "unphysical" field $\mathbf{B}_{1 \mathrm{D}}^{u}=\left(0,0, B_{1} z\right)$ which is considered in most standard treatments but does not satisfy $\boldsymbol{\nabla} \cdot \mathbf{B}=0$, and a "physical" field $\mathbf{B}_{1 \mathrm{D}}^{p}=\left(0,0, B_{1} x\right)$ which satisfies $\boldsymbol{\nabla} \cdot \mathbf{B}=0$. These two fields are shown in Fig. 1 with $B_{1}$ indicating the inhomogeneity strength. Both fields point in the $z$ direction; their inhomogeneities in $z$ and in $x$, respectively.

The Hamiltonians corresponding to these two fields are given by

$$
H_{1 \mathrm{D}}^{u}=\frac{p_{x}^{2}+p_{z}^{2}}{2 m}-\mu B_{1} z \sigma_{z}, \quad H_{1 \mathrm{D}}^{p}=\frac{p_{x}^{2}+p_{z}^{2}}{2 m}-\mu B_{1} x \sigma_{z} .
$$

We can construct the spin-1/2 propagators $K\left(x, x_{0}, z, z_{0} ; t\right)$ from the one-dimensional spinless propagator for linear potential corresponding to a Hamiltonian

$$
H=\frac{p_{x}^{2}}{2 m}+f x
$$

where $f$ is constant. To construct this propagator we apply an algebraic method based on the recognition of a group algebra [29]. The linear potential propagator can be obtained in a straightforward way by relating $\left[\partial_{x}^{2}, x\right]=2 \partial_{x}$ to $\left[a^{2}, a^{\dagger}\right]=2 a$ through the substitution $\partial_{x} \rightarrow a$ and $x \rightarrow a^{\dagger}$. While the exponent in a time-evolution operator $\exp \left(-\frac{i H t}{\hbar}\right)$ involves noncommuting operators $a$ and $a^{\dagger}$, Katriel's formula [39]

$$
\exp \left(\alpha a^{\dagger}+\beta a^{r}\right)=e^{\alpha a^{\dagger}} \exp \left[\sum_{i=0}^{r} \beta \alpha^{i}\left(\begin{array}{l}
r \\
i
\end{array}\right) \frac{1}{1+i} a^{r-i}\right]
$$

applied to $r=2$ makes a separation of the kinetic energy and potential energy term inside the time-evolution operator possible. After some algebraic manipulations, the one-dimensional linear potential propagator [34] is recovered:

$$
\begin{aligned}
& K\left(x, x_{0} ; t\right) \\
& \quad=\sqrt{\frac{m}{2 \pi i \hbar t}} \exp \left(-\frac{m\left(x-x_{0}\right)^{2}}{2 i \hbar t}+\frac{f\left(x+x_{0}\right) t}{2 i \hbar}+\frac{f^{2} t^{3}}{24 i \hbar m}\right) .
\end{aligned}
$$

We now proceed to construct the spin-1/2 propagators based on Eq. (5). It should be noted that in the 1D-inhomogeneity case, $\sigma_{z}$ acts as a place holder for its diagonal elements $(+/-)$. Therefore, the potential $f x$ for spinless particles can be replaced by $\sigma_{z} f x$ for spin- $1 / 2$ particles. With this replacement, Katriel's formula is still valid since $\left[\sigma_{i}, \sigma_{j}\right]=2 i \epsilon_{i j k} \sigma_{k}$ and $\left[\sigma_{z}, \sigma_{z}\right]=0$. The constant $f$ corresponds to the magnetic term $-\mu B_{1} \sigma_{z}$ in both unphysical and physical cases. As a result, the propagator $K_{1 \mathrm{D}}^{u}\left(z, z_{0} ; t\right)$ for the unphysical field $B_{1 \mathrm{D}}^{u}$ and the propagator $K_{1 \mathrm{D}}^{p}\left(x, x_{0} ; t\right)$ for the physical field $B_{1 \mathrm{D}}^{p}$ are given in one dimension by

$$
\begin{aligned}
K_{1 \mathrm{D}}^{u}\left(z, z_{0} ; t\right) & =\sqrt{\frac{m}{2 \pi i \hbar t}} \exp \left(-\frac{m\left(z-z_{0}\right)^{2}}{2 i \hbar t}-\frac{\mu B_{1} \sigma_{z}\left(z+z_{0}\right) t}{2 i \hbar}+\frac{\mu^{2} B_{1}^{2} t^{3}}{24 i \hbar m}\right), \\
K_{1 \mathrm{D}}^{p}\left(x, x_{0} ; t\right) & =\sqrt{\frac{m}{2 \pi i \hbar t}} \exp \left(-\frac{m\left(x-x_{0}\right)^{2}}{2 i \hbar t}-\frac{\mu B_{1} \sigma_{z}\left(x+x_{0}\right) t}{2 i \hbar}+\frac{\mu^{2} B_{1}^{2} t^{3}}{24 i \hbar m}\right) .
\end{aligned}
$$

For $\mu B_{1}=0$, Eqs. (6) and (7) reduce to the free particle propagator

$$
K^{\text {free }}\left(x, x_{0} ; t\right)=\sqrt{\frac{m}{2 \pi i \hbar t}} \exp \left(-\frac{m\left(x-x_{0}\right)^{2}}{2 i \hbar t}\right) .
$$

The free propagator includes quantum-mechanical spreading, a feature which will also be observed in the presence of external fields.
For 1D inhomogeneities, each dimension is independent. The propagator can then be expressed as

$$
K\left(x, z, x_{0}, z_{0} ; t\right)=K\left(x, x_{0} ; t\right) K\left(z, z_{0} ; t\right) .
$$

As a result, one can extend Eqs. (6) and (7) to

$$
\begin{aligned}
& K_{1 \mathrm{D}}^{u}\left(x, z, x_{0}, z_{0} ; t\right)=\frac{m}{2 \pi i \hbar t} \exp \left(-\frac{\left(x-x_{0}\right)^{2}+\left(z-z_{0}\right)^{2}}{2 i \hbar t / m}-\frac{\mu B_{1} \sigma_{z}\left(z+z_{0}\right) t}{2 i \hbar}+\frac{\mu^{2} B_{1}^{2} t^{3}}{24 i \hbar m}\right), \\
& K_{1 \mathrm{D}}^{p}\left(x, z, x_{0}, z_{0} ; t\right)=\frac{m}{2 \pi i \hbar t} \exp \left(-\frac{\left(x-x_{0}\right)^{2}+\left(z-z_{0}\right)^{2}}{2 i \hbar t / m}-\frac{\mu B_{1} \sigma_{z}\left(x+x_{0}\right) t}{2 i \hbar}+\frac{\mu^{2} B_{1}^{2} t^{3}}{24 i \hbar m}\right),
\end{aligned}
$$



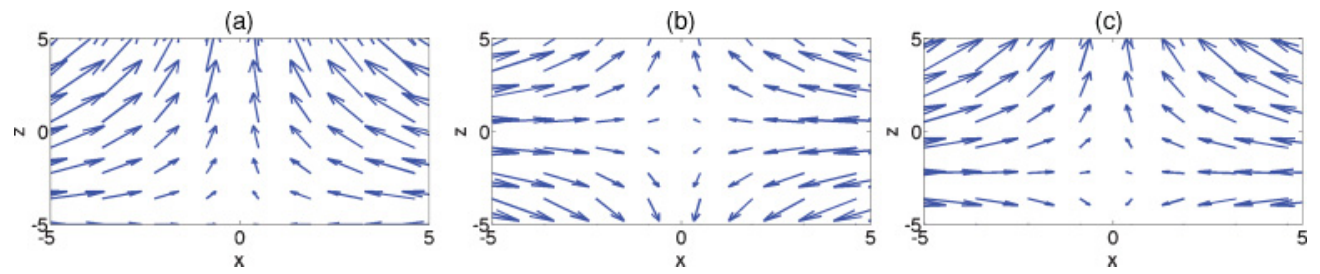

FIG. 2. (Color online) Plots of the magnetic field $\mathbf{B}=\left(-B_{1} x, 0, B_{1} z+B_{0}\right)$ with different inhomogeneity strengths $B_{1}$ and homogeneous fields $B_{0}$. (a) $B_{1}=1.0, B_{0}=5.0$, (b) $B_{1}=1.0, B_{0}=0.0$, (c) $B_{1}=2.0, B_{0}=5.0$.

where the free particle propagator of Eq. (8) is used for the dimensions where no inhomogeneity is present.

The result of Eq. (10) disagrees with the (2D) expression of Eq. (12) found in [24] by both the universal signature factor $\frac{\mu^{2} B_{1}^{2} t^{3}}{24 i \hbar m}$ for the linear potential and the term linear in $t$.

$$
\begin{aligned}
& K_{\mathrm{SSM}}=\frac{m}{i \hbar t} \exp \left(-\frac{m\left(x-x_{0}\right)^{2}}{2 i \hbar t}-\frac{m\left(z-z_{0}-\frac{\mu B_{0} \sigma_{z} t^{2}}{2 m}\right)}{2 i \hbar t}\right), \\
& K_{\mathrm{SLB}} \\
& \quad=\eta(t) \exp \left\{\frac{m}{\hbar t}\left[\left(z-z_{0}-\frac{\mu \sigma_{z} B_{1} t^{2}}{2 m}\right)^{2}-\frac{2 \hbar \mu B_{1} t^{2} z \sigma_{z}}{m}\right]\right\},
\end{aligned}
$$

where the subscripts SSM and SLB refer to the authors of reference [24] Scully, Shea, and McCullen, and reference [25] Scully, Lamb, and Barut, respectively. This same result also differs from the expression found in Eq. (13) [25] by the factor $\frac{\mu^{2} B^{2} t^{3}}{24 i \hbar m}$. One can check that $K$ in Eq. (10) satisfies the Pauli-Schrödinger equation. The corresponding expressions for Eq. (12) in [24] and Eq. (13) in [25] do not.

Once the propagator $K$ is constructed, the wave packet evolution $\psi(x, t)$ can be obtained by applying the propagator to an initial wave packet $\psi\left(x_{0}, 0\right)$, i.e.,

$$
\psi(x, t)=\int_{-\infty}^{\infty} K\left(x, x_{0} ; t\right) \psi\left(x_{0}, 0\right) d x_{0} .
$$

To show the localization effect in the SGE, we choose $\psi\left(x_{0}, z_{0}, 0\right)$ to be a spinor with Gaussian distribution in space centered at $\left(x_{0}, z_{0}\right)=\left(x^{\prime}, z^{\prime}\right)$ and with widths in two dimensions $w_{x}$ and $w_{z}$, such that $\psi\left(x_{0}, z_{0}, 0\right)=\frac{1}{\sqrt{\pi w_{x} w_{z}}} \exp \left(-\frac{\left(x_{0}-x^{\prime}\right)^{2}}{2 w_{x}^{2}}-\right.$ $\left.\frac{\left(z_{0}-z^{\prime}\right)^{2}}{2 w_{z}^{2}}\right)(\alpha|\uparrow\rangle+\beta|\downarrow\rangle)$, where $|\uparrow\rangle$ and $|\downarrow\rangle$ are up-in- $z$ and down-in- $z$ spin states with constant coefficients $\alpha$ and $\beta$ chosen to satisfy $|\alpha|^{2}+|\beta|^{2}=1$. Thus we consider a product state (unentangled) of space and spin. Note that the choice of initial positions $\left(x_{0}, z_{0}\right)$ will lead to the appearance of different dynamics. In addition to the coherent states, we can also consider mixed states such as the mixture of $50 \%$ spin-up and $50 \%$ spin-down propagates. Because of the linearity of the Schrödinger equation and of the propagator, the method can be applied to spinors with different spatial localizations for spinup and spin-down particles (nonproduct or entangled states). In particular it can be applied repeatedly for arbitrary times in spin-separating dynamics without further modifications.

\section{B. 2D-inhomogeneity propagation}

For fields with inhomogeneities in two dimensions (2D inhomogeneity), complexity arises from the noncommutativity among Pauli matrices, namely, $\left[\sigma_{i}, \sigma_{j}\right]=2 i \epsilon_{i j k} \sigma_{k}$. This leads to position-dependent eigenspinors, in contrast to the global eigenspinors of the 1D case. The nonexistence of global eigenspinors leads to a richer spin dynamics.

In Fig. 2 we consider magnetic fields with 2D inhomogeneity. The values of the homogeneous component $B_{0}$ and the magnitude of the inhomogeneity $B_{1}$ can be chosen so as to move the saddle [40] point $(0,-5)$ in Fig. 2(a), $(0,0)$ in Fig. 2(b), and $(0,-2.5)$ in Fig. 2(c). Figure 2(b), which corresponds to $\mathbf{B}=\left(-B_{1} x, 0, B_{1} z\right)$, is typically used when treating the $2 \mathrm{D}$-inhomogeneity problem in the Stern-Gerlach experiment. The Stern-Gerlach magnetic field corresponds to regions of the upper-half of Fig. 2(b). Therefore, the Stern-Gerlach setup can be described analytically by a beam located at $(0,0)$ in a field with a large homogeneous component $B_{0}$ leading to $\mathbf{B}=\left(-B_{1} x, 0, B_{0}+B_{1} z\right)$ as in Fig. 2(a) or by a beam located in the upper-half plane of Fig. 2(b) without the use of a homogeneous component. This is the choice used below when we select an initial wave packet centered around $z=z_{0}$ with $z_{0}>0$. We thus cover the historical Stern-Gerlach case by staying away from the saddle point in the field. Later we will consider what happens when the beam extends beyond the transition axis. This transition axis goes through the saddle point and is perpendicular to the radial vector connecting the center of the initial packet with the saddle point. The radial vector corresponds to the direction of steepest gradient of the inhomogeneous field in the center of the packet. Notice that the dynamics in the region around the saddle point is of great interest when considering a quadruple field, such as produced with anti-Helmholtz coils. This is relevant to the physics of magneto-optical traps (MOTs).

The construction of analytic Stern-Gerlach propagators in $2 \mathrm{D}$ inhomogeneities is challenging. Note that even with approximated analytic propagators, the application of the integral formula Eq. (14) can still be a daunting task. In order to evaluate the evolution of wave packets in a more efficient way, we use the Trotter product formula [38] for noncommuting operators $a_{1}$ and $a_{2}$ applied directly on the wave packet:

$$
\begin{aligned}
\exp [ & \left.-\tau\left(a_{1}+a_{2}\right)\right] \psi\left(x_{0}, 0\right) \\
\approx & \lim _{n \rightarrow \infty}\left[\exp \left(-\frac{\tau}{2 n} a_{1}\right) \exp \left(-\frac{\tau}{n} a_{2}\right) \exp \left(-\frac{\tau}{2 n} a_{1}\right)\right]^{n} \\
& \times \psi\left(x_{0}, 0\right) .
\end{aligned}
$$

This improves upon the simpler assumption

$$
\exp \left[-\frac{\tau}{n}\left(a_{1}+a_{2}\right)\right] \approx \exp \left(-\frac{\tau}{n} a_{1}\right) \exp \left(-\frac{\tau}{n} a_{2}\right)
$$

for large $n$. We set $a_{1}$ to be the potential energy term and $a_{2}$ to be the kinetic energy term. Instead of using the propagator 
integral formula, we apply the time-evolution operator directly to the wave packet. The Trotter product formula (15) allows us to operate on the wave packet repeatedly with a reasonable value of $n$. Since both the wave packet and the potential $\left(a_{1}\right)$ are in the coordinate representation, the operation is purely multiplicative. Before applying the kinetic operator $\left(a_{2}\right)$ to the wave function, it is most convenient to perform a fast Fourier transform on all grid points. After applying the kinetic operator, we perform a fast inverse Fourier transform on all (momentum) grid points. This approach is an application of the general split-step (Fourier) method, also used in molecular dynamics [41]. It has the advantage of preserving unitarity. Once we recover the evolved wave packet in the coordinate representation for a time interval $\tau$, we generate spin density plots by projecting wave packets in specific directions in spin space. Note that in order for Eq. (15) to hold, one needs to set $\tau / n$ small enough (or $n$ large enough). This condition can be verified through the stability of the results as $n$ increases. The region in space also needs to be chosen large enough to avoid contamination from the artificial periodic boundary introduced by the fast Fourier transform.

\section{RESULTS}

\section{A. Spin densities for pure and mixed states}

We obtain $\psi(x, z ; t)$ by propagating $\psi(x, z ; 0)$. Local spin densities $\langle\mathbf{S}\rangle(x, z)=\langle\psi(x, z ; t)|\mathbf{S}| \psi(x, z ; t)\rangle$ can then be evaluated at all times. The brackets refer to an integration (summation) over spin variables but not over coordinate space. We will discuss and interpret features in the local spin densities $\langle\mathbf{S}\rangle$ that represent the appearance of a characteristic spin dynamics structure for both 1D and 2D inhomogeneities in the magnetic field. In both cases, we label spin states using a subscripted arrow convention. In particular, up-in- $x$ $\left(|\uparrow\rangle_{x}\right)$ and down-in- $x\left(|\downarrow\rangle_{x}\right)$ spin states correspond to balanced superpositions of up-in- $z(|\uparrow\rangle)$ and down-in- $z(|\downarrow\rangle)$ spin states

$$
|\uparrow\rangle_{x}=\frac{|\uparrow\rangle+|\downarrow\rangle}{\sqrt{2}}, \quad|\downarrow\rangle_{x}=\frac{|\uparrow\rangle-|\downarrow\rangle}{\sqrt{2}},
$$

and similarly for up-in- $y\left(|\uparrow\rangle_{y}\right)$ or down-in- $y\left(|\downarrow\rangle_{y}\right)$ spin states

$$
\begin{aligned}
|\uparrow\rangle_{y}= & \frac{|\uparrow\rangle+i|\downarrow\rangle}{\sqrt{2}}, \quad|\downarrow\rangle_{y}=\frac{|\uparrow\rangle-i|\downarrow\rangle}{\sqrt{2}} . \quad \begin{array}{l}
\text { and } \psi_{\downarrow 1 D}^{u}(x, z ; t) \text { corresponding to spin-up and spin-down } \\
\text { in } z:
\end{array} \\
\psi_{\uparrow 1 D}^{u}(x, z ; t)= & \frac{\alpha}{\sqrt{\pi w_{x} w_{z}} \sqrt{\left(1+\frac{i \hbar t}{m w_{x}^{2}}\right)\left(1+\frac{i \hbar t}{m w_{z}^{2}}\right)}} \\
& \times \exp \left(-\frac{m\left[x^{2}+z^{2}+\frac{i \hbar t}{m}\left(x^{\prime 2}+z^{\prime 2}\right)\right]}{2 i \hbar t}+\frac{m\left(x+\frac{i \hbar t x^{\prime}}{m w_{x}^{2}}\right)^{2}}{2 i \hbar t\left(1+\frac{i \hbar t}{m w_{x}^{2}}\right)}+\frac{m\left(z+\frac{i \hbar t z^{\prime}}{m w_{z}^{2}}-\frac{\mu B_{1} t^{2}}{2 m}\right)^{2}}{2 i \hbar t\left(1+\frac{i \hbar t}{m w_{z}^{2}}\right)}-\frac{\mu B_{1} t z}{2 i \hbar}+\frac{\mu^{2} B_{1}^{2} t^{3}}{24 i \hbar m}\right) \\
\psi_{1 \mathrm{D}}^{\downarrow u}(x, z ; t)= & \left.\frac{\beta}{\left(19 w_{x}\right.}\right) \\
& \times \exp \left(-\frac{m\left[x^{2}+z^{2}+\frac{i \hbar t}{m}\left(x^{\prime 2}+z^{\prime 2}\right)\right]}{2 i \hbar t}+\frac{m\left(x+\frac{i \hbar t x^{\prime}}{m w_{x}^{2}}\right)^{2}}{2 i \hbar t\left(1+\frac{i \hbar t}{m w_{x}^{2}}\right)}+\frac{m\left(z+\frac{i \hbar t z^{\prime}}{m w_{z}^{2}}+\frac{\mu B_{1} t^{2}}{2 m}\right)^{2}}{2 i \hbar t\left(1+\frac{i \hbar t}{m w_{z}^{2}}\right)}+\frac{\mu B_{1} t z}{2 i \hbar}+\frac{\mu^{2} B_{1}^{2} t^{3}}{24 i \hbar m}\right) .
\end{aligned}
$$

In the simulations, we analyze the spin dynamics for various parameter values: we specify the initial location of the wave packet centered around $\left(x^{\prime}, z^{\prime}\right)$, the interaction strength $\mu B_{1}$, the widths of the Gaussian wave packet $\left(w_{x}, w_{z}\right)$, and the time interval $t$ over which we follow the dynamics.

We choose to combine the magnetic moment and the field into one entity $\mu B_{1}$, because it is the product of $\mu$ and $B_{1}$ that gives the strength of the effect. The combination $\mu B_{1}$ is a universal Stern-Gerlach "interaction strength," against which different magnetic fields can be chosen for atomic systems with different magnetic moments. It plays a similar role to the spinorbit Rashba interaction strength in condensed matter systems $[35,37]$. We provide results in natural units $\hbar=1, m=1$. Note also that since we are interested in the influence of the width on the dynamics, we select an absolute length unit $d$ such that the width $w$ and the positions $x$ and $y$ are all expressed in terms of $d$ rather than being correlated as a result of the choice of units. We also provide the units for the following variables: $\mu B_{1}$ is in units of $\hbar^{2} / m d^{3}$ while $t$ is in units of $m d^{2} / \hbar$. This allows us to recover experimentally accessible values of the magnetic field strength $B_{1}$ by substituting realistic values of $\hbar$ and $m$ and by choosing an appropriate length unit $d$. This also determines a unit width and a unit time and therefore realistic orders of magnitude for time and for wave packet width.

Finally we also compare results of $\langle\mathbf{S}\rangle$ for an initial pure state $\left(\frac{|\uparrow\rangle+|\downarrow\rangle}{\sqrt{2}}\right)$ (coherent state or C-state) and for a totally mixed state consisting of $50 \%|\uparrow\rangle$ and $50 \%|\downarrow\rangle$ (incoherent state or IC-state) spin states. We select the C-state (totally polarized) and IC-state (totally unpolarized) to maximize the effect of polarization. It turns out that a Stern-Gerlach separation is the same for the C-state and the IC-state when spin flipping is not possible. Conversely we also show that the C-state and the IC-state display different features in a magnetic field with $2 \mathrm{D}$ inhomogeneities, when spin flipping can occur.

\section{B. Spin-evolution features for 1D inhomogeneity}

After applying the propagator $K_{1 \mathrm{D}}^{u}$ in Eq. (10) to an initial spin wave packet $\psi(x, z ; 0)$, we obtain an evolved spin wave packet $\psi_{1 \mathrm{D}}^{u}(x, z ; t)$ consisting of two components $\psi_{\uparrow 1 D}^{u}(x, z ; t)$ 
Applying $K_{1 \mathrm{D}}^{p}$ in Eq. (11) to $\psi(x, z ; 0)$ similarly gives the two components

$$
\begin{aligned}
\psi_{\uparrow 1 \mathrm{D}}^{p}(x, z ; t)= & \frac{\alpha}{\sqrt{\pi w_{x} w_{z}} \sqrt{\left(1+\frac{i \hbar t}{m w_{x}^{2}}\right)\left(1+\frac{i \hbar t}{m w_{z}^{2}}\right)}} \\
& \times \exp \left(-\frac{m\left[x^{2}+z^{2}+\frac{i \hbar t}{m}\left(x^{\prime 2}+z^{\prime 2}\right)\right]}{2 i \hbar t}+\frac{m\left(z+\frac{i \hbar t z^{\prime}}{m w_{z}^{2}}\right)^{2}}{2 i \hbar t\left(1+\frac{i \hbar t}{m w_{z}^{2}}\right)}+\frac{m\left(x+\frac{i \hbar t x^{\prime}}{m w_{x}^{2}}-\frac{\mu B_{1} t^{2}}{2 m}\right)^{2}}{2 i \hbar t\left(1+\frac{i \hbar t}{m w_{x}^{2}}\right)}-\frac{\mu B_{1} t x}{2 i \hbar}+\frac{\mu^{2} B_{1}^{2} t^{3}}{24 i \hbar m}\right) \\
\psi_{\downarrow 1 \mathrm{D}}^{p}(x, z ; t)= & \frac{\beta}{\sqrt{\pi w_{x} w_{z}} \sqrt{\left(1+\frac{i \hbar t}{m w_{x}^{2}}\right)\left(1+\frac{i \hbar t}{m w_{z}^{2}}\right)}} \\
& \times \exp \left(-\frac{m\left[x^{2}+z^{2}+\frac{i \hbar t}{m}\left(x^{\prime 2}+z^{\prime 2}\right)\right]}{2 i \hbar t}+\frac{m\left(z+\frac{i \hbar z^{\prime}}{m w_{z}^{2}}\right)^{2}}{2 i \hbar t\left(1+\frac{i \hbar t}{m w_{z}^{2}}\right)}+\frac{m\left(x+\frac{i \hbar t x^{\prime}}{m w_{x}^{2}}+\frac{\mu B_{1} t^{2}}{2 m}\right)^{2}}{2 i \hbar t\left(1+\frac{i \hbar t}{m w_{x}^{2}}\right)}+\frac{\mu B_{1} t x}{2 i \hbar}+\frac{\mu^{2} B_{1}^{2} t^{3}}{24 i \hbar m}\right) .
\end{aligned}
$$

With these evolved solutions we construct the spin densities $\langle\mathbf{S}\rangle(x, z)$ and display the results as $2 \mathrm{D}$ contour plots (Fig. 3) and cuts in selected directions as explained below. From the displayed spin density, we observe three interesting features: a spin-separating mechanism (SSM), a bamboo-shooting structure (BSS), and a persistent spin helix (PSH). Note that BSS and PSH are seen only for a coherent beam, whereas SSM appears in both the coherent and the incoherent cases. Note that the plots of the features provided here are only selected snapshots. The display of successive snapshots in time leads to spin-evolution animations.

\section{Spin-separating mechanism (SSM)}

The spin-separating mechanism occurs when a homogeneous spin density develops into an inhomogeneous spin density, with different spin components occurring in different regions of space. We observe the spin-separation mechanism in the plots of $\left\langle S_{z}\right\rangle$ in Fig. 3. In the 1D case, $S_{z}$ commutes with the Hamiltonian and their common eigenvectors are global or position independent. The $\mathbf{B}_{1 \mathrm{D}}^{u}$ field leads to the textbook Stern-Gerlach effect with vertical separation [Fig. 3(a)], while $\mathbf{B}_{1 \mathrm{D}}^{p}$ leads a horizontal spin separation [Fig. 3(b)]. Both cases exhibit entanglement of spin and space.

(a)

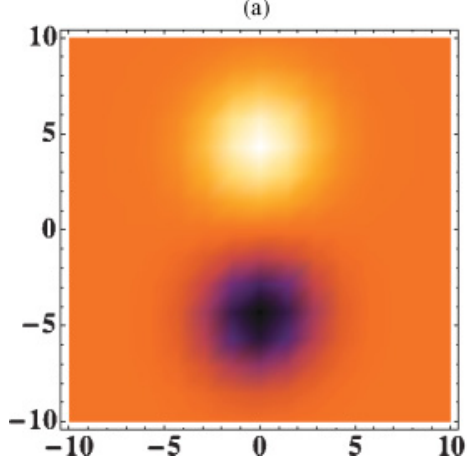

From Fig. 3, the eigenspinors, namely, $|\uparrow\rangle$ and $|\downarrow\rangle$ spin states, get separated in the direction of the inhomogeneity.

Note that in Fig. 3 for the $\mathbf{B}_{1 \mathrm{D}}^{u}\left(\mathbf{B}_{1 \mathrm{D}}^{p}\right)$, the $x(z)$ dimension is not important. The effect of separation can also be seen clearly by limiting one's attention to the inhomogeneity axis by setting $x=0(z=0)$. In Fig. 4 , we display the effect of interaction strength $\mu B_{1}$, and of the width of the packet $w$ on the rate of separation. We plot the spin density $\left\langle S_{z}\right\rangle$ along the central inhomogeneity axis $\left(x=0\right.$ for $\mathbf{B}_{1 \mathrm{D}}^{u}$ or $z=0$ for $\mathbf{B}_{1 \mathrm{D}}^{p}$ ) in Fig. 4, where the horizontal axis refers to the $z$ (for $\mathbf{B}_{1 \mathrm{D}}^{u}$ ) or $x$ (for $\mathbf{B}_{1 \mathrm{D}}^{p}$ ) dimension. In Fig. 4, faster separation is observed for increased interaction strength $\mu B_{1}$. This is consistent with the semiclassical interpretation, where the force is proportional to the gradient of the field for the same particle. Reducing the width of the initial packet leads to faster spreading and to a decrease of the amplitude as expected from complementarity and quantum localization. In the plots, we choose to vary $w_{i}$, the width along the inhomogeneity axis only. Reducing the width in the perpendicular direction only leads to an overall decrease of the amplitude in this section's plot. Note that the results are the same for both the C-state $\frac{|\uparrow\rangle+|\downarrow\rangle}{2}$ and the IC-state $(50 \%|\uparrow\rangle$ and $50 \%|\downarrow\rangle)$. This can be understood because no spin flippings would occur. (b)

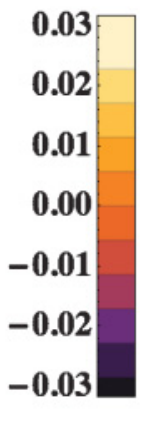

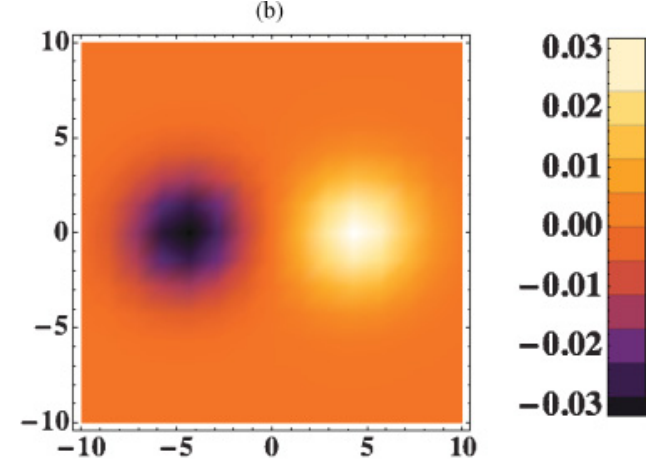

FIG. 3. (Color online) Contour plots of spin density $\left\langle S_{z}\right\rangle(x, z)$ for an initial spin state $|\uparrow\rangle_{x}$ centered around $\left(x^{\prime}, z^{\prime}\right)=(0,0)$ for the interaction strength $\mu B_{1}=0.1$ (in units of $\hbar^{2} / m d^{3}$ ), wave packet widths $w_{x}=w_{z}=1$ (in units of $d$ ), and time $t=5$ (in units of $m d^{2} / \hbar$ ) in two possible magnetic fields. (a) $\mathbf{B}_{1 d}^{u}=\left(0,0, B_{1} z\right)$, (b) $\mathbf{B}_{1 d}^{p}=\left(0,0, B_{1} x\right)$. 


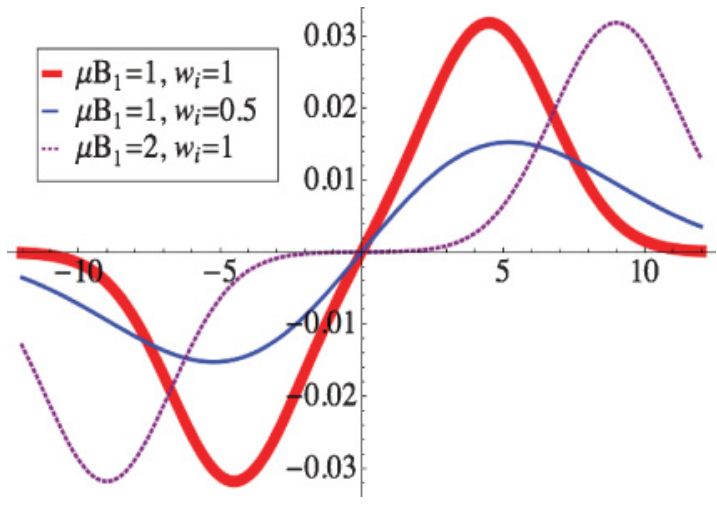

FIG. 4. (Color online) Spin density $\left\langle S_{z}\right\rangle$ as a function of position in the inhomogeneity direction ( $z$ for $\mathbf{B}_{1 \mathrm{D}}^{u}$ or $x$ for $\mathbf{B}_{1 \mathrm{D}}^{p}$ ) for an initial spin state $|\uparrow\rangle_{x}$ centered around $\left(x^{\prime}, z^{\prime}\right)=(0,0)$ at $t=3$ (in units of $m d^{2} / \hbar$ ) with different sets of interaction strengths $\mu B_{1}$ (in units of $\hbar^{2} / m d^{3}$ ) and wave packet widths in the inhomogeneity direction $w_{i}$ (in units of $d$ ).

\section{Bamboo-shooting structure (BSS)}

BSS represents the successive rise of spin polarization at fixed interval along the axis of inhomogeneity. One sees BSS in Fig. 5 when observing the spin projected in a direction not along the eigenspinors of the system, in particular $\left\langle S_{x}\right\rangle$ and $\left\langle S_{y}\right\rangle$. In Fig. 5, we plot the local spin density $\left\langle S_{x}\right\rangle$ along the inhomogeneity direction.

The BSS can be observed for initial C-states $|\uparrow\rangle_{x}$ and $|\uparrow\rangle_{y}$, which are both superpositions of eigenspinors $|\uparrow\rangle$ and $|\downarrow\rangle$. For IC-states, such as $50 \%|\uparrow\rangle$ and $50 \%|\downarrow\rangle$, one does not observe BSS. Since the system does not induce spin flippings for eigenspinors, the final spin polarization remains unchanged and $\left\langle S_{x}\right\rangle=0$ applies at all times in this case.

The spin density $\left\langle S_{x}\right\rangle$ is generated for cases where one can manipulate the interaction strength $\mu B_{1}$ and the width of the wave packet $w_{i}$. The parameters $\mu B_{1}$ and $w_{i}$ are chosen for visual readability. The wave packet extends farther as time evolves as a result of quantum spreading. We also observe oscillatory motion for the noneigenspinors. The period of the

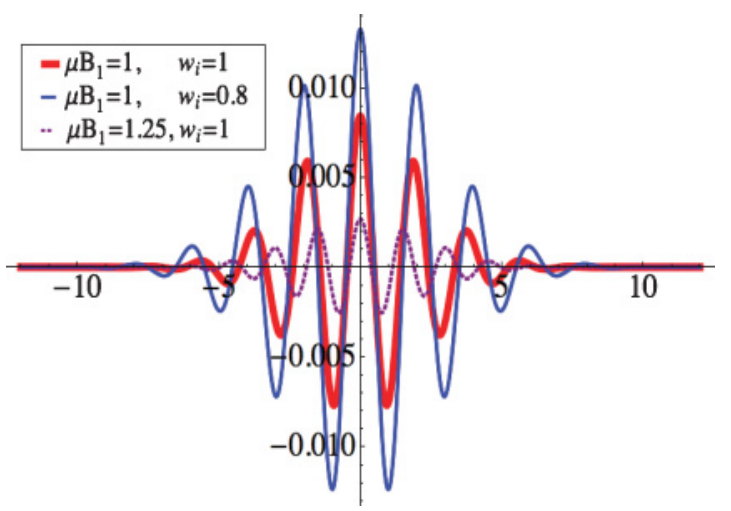

FIG. 5. (Color online) Spin density $\left\langle S_{x}\right\rangle$ as a function of position in the inhomogeneity direction ( $z$ for $\mathbf{B}_{1 \mathrm{D}}^{u}$ or $x$ for $\mathbf{B}_{1 \mathrm{D}}^{p}$ ) for an initial spin state $|\uparrow\rangle_{x}$ centered around $\left(x^{\prime}, z^{\prime}\right)=(0,0)$ at $t=3$ (in units of $\left.m d^{2} / \hbar\right)$ with different sets of interaction strength $\mu B_{1}$ (in units of $\hbar^{2} / m d^{3}$ ) and wave packet width in the inhomogeneity direction $w_{i}$ (in units of $d$ ). oscillation decreases as one increases the interaction strength $\mu B_{1}$. For an initial C-state $\left(|\uparrow\rangle_{x}\right)$, one has both $|\uparrow\rangle$ and $|\downarrow\rangle$ spin components. As discussed previously, the two components separate faster with larger $\mu B_{1}$. This leads to less overlap between the components, which results in overall decrease of the amplitude. One also notices that as the eigenspinors move more rapidly, the noneigenspinors would experience oscillation with an increase in the frequency or a decrease in the period. The period increases when the width of the wave packet is reduced in the direction of inhomogeneity in Fig. 5.

It is noteworthy to mention that Griffiths [16] argues that the operator $S_{x}$ oscillates rapidly in the Heisenberg picture and averages to zero to justify the argument that the second inhomogeneity is not important. In a way, the BSS feature confirms the rapid oscillatory and zero-averaging behavior of $S_{x}$. It is interesting to note that in the case where two inhomogeneities are present, as we discuss in Sec. IIIC, we find that the argument used to neglect the second inhomogeneity is no longer valid. This paradox can be explained by the lack of constants of the motion for any spin operators in the 2D-inhomogeneity case.

\section{Persistent spin helix (PSH)}

Persistent spin helix refers to the precessional motion of the spin in the $x y$ plane. One observes a PSH structure by generating the spin densities $\left\langle S_{x}\right\rangle$ and $\left\langle S_{y}\right\rangle$ starting from a coherent state $|\uparrow\rangle_{x}$. This effect can only be observed from a C-state in the noneigenspinor basis [up-in- $x(y)$, down-in$x(y)]$. In Fig. 6, we observe that $\left\langle S_{x}\right\rangle$ exhibits even symmetry, while $\left\langle S_{y}\right\rangle$ exhibits odd symmetry with respect to the original location of the packet. The $\left\langle S_{x}\right\rangle$ is shifted with respect to $\left\langle S_{y}\right\rangle$. By following the spin component on the $z$ axis, one sees that a persistent spin helix forms with clockwise motion to the right. As discussed in the BSS section, the period of the helix can be controlled by manipulating the interaction strength $\mu B_{1}$ and wave packet width in the inhomogeneity direction $w_{i}$. Therefore one can control the spin polarization at specific locations by manipulating either the interaction strength $\mu B_{1}$ or the wave packet width in the inhomogeneity direction $w_{i}$. This mechanism allows one to construct structures with controlled spin densities in the sense of SG engineering.

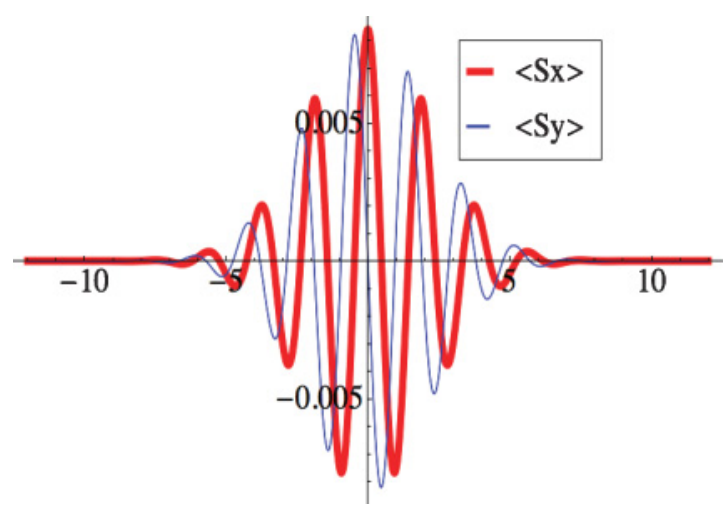

FIG. 6. (Color online) Plot of spin densities $\left\langle S_{x}\right\rangle$ and $\left\langle S_{y}\right\rangle$ at $t=3$ (in units of $m d^{2} / \hbar$ ) with $\mu B_{1}=1$ (in units of $\hbar^{2} / m d^{3}$ ) and $w_{i}=1$ (in units of $d$ ) where the axis refers to the position in the inhomogeneity direction ( $z$ for $\mathbf{B}_{1 \mathrm{D}}^{u}$ or $x$ for $\mathbf{B}_{1 \mathrm{D}}^{p}$ ). 


\section{Spin-evolution features for 2D inhomogeneity}

We now display evolved wave packets in systems with 2D inhomogeneity. Due to the continuous evolving nature of the wave packet in the $2 \mathrm{D}$ case, plots are generated at different times to show explicit features individually. The plots are all two dimensional and show contours of equal spin densities.

From the spin density contour plots, one observes three interesting features: radial spin separation (RSS), asymmetryinduced contamination (AIC), and four-lobes structure (FLS). Note that RSS and FLS are observed with an initial spin state $|\uparrow\rangle_{x}$ and $50 \%|\uparrow\rangle$ and $50 \%|\downarrow\rangle$ whereas AIC is observed only for an initial C-state $|\uparrow\rangle$.

\section{Radial spin separation (RSS)}

Plots of the spin density $\left\langle S_{z}\right\rangle$ are generated in a field in Fig. 2(b) for the initial C-state $|\uparrow\rangle_{x}$ in Fig. 7 and for the IC-state $(50 \%|\uparrow\rangle$ and $50 \%|\downarrow\rangle)$ in Fig. 8. In both cases the initial wave packet centered around $\left(x^{\prime}, z^{\prime}\right)=(0,4)$. Both cases exhibit textbook Stern-Gerlach effect: vertical spin separation of $|\uparrow\rangle$ and $|\downarrow\rangle$. In both cases, the rate of separation increases when one increases the interaction strength $\mu B_{1}$. By reducing the width in the $x$ direction, the wave packet spreads faster in $x$.

Comparing the initial C-state with the initial IC-state, we notice that IC-states show $x \rightarrow-x$ symmetry, whereas C-states do not. At any particular time, the plots of C-state wave packets show an $x \rightarrow-x$ asymmetry. This may seem surprising given the symmetric nature of the field in Fig. 2. In fact these plots in Fig. 2 are incomplete since they do not incorporate the spin direction and therefore miss the asymmetry in the $\mu \cdot \mathbf{B}$ interaction term. The $x$ symmetry is restored in the balanced spin mixture of the IC-state. The consistency of this asymmetry can be checked in the following way: (i) By performing a reflection about the $z$ axis only, namely, $x \rightarrow-x$, in the field configuration, we observe that the oscillation is still present but its direction is reversed. Note that the field becomes unphysical under such transformation $B \rightarrow B^{\prime}=(x, 0, z)$. (ii) By changing the initial spin state $\left(|\uparrow\rangle_{x} \rightarrow|\downarrow\rangle_{x}\right)$ only, we observe that the oscillation is still present but its direction is reversed. (iii) By performing both a reflection about the $z$ axis in the field configuration and a reversal of the initial spin state, we observe that the oscillation direction is unchanged. (iv) By switching the dependence on $x$ and $z$, namely, by starting with $\mathrm{a}|\uparrow\rangle$ spin state centered around $\left(x^{\prime}, z^{\prime}\right)=(4.0,0.0)$, we observe that the oscillation in time now displays a vertical asymmetry.

From these observations, we conclude that a C-state, which is a superposition of eigenstates centered around a noneigenspinor axis experiences two mechanisms: spin separation and oscillations in time about the second inhomogeneity axis. For example, this can happen for both a $|\uparrow\rangle_{x}$ spin state centered around the $z$ axis and a $|\uparrow\rangle$ spin state centered around the $x$ axis. Also, this oscillation is short-lived if the wave packet is placed farther away from the saddle point. This shows that the oscillation depends on the degree to which the two eigenstates overlap, for example, $|\uparrow\rangle$ and $|\downarrow\rangle$ on the $z$ axis. A complete spin separation is more easily obtained when an initial state enters the field far away from the saddle point. This can be understood as follows: In an analysis of wave packet dynamics, it is unavoidable that one has to take the spreading of the wave packet into account. The spreading induces spin flippings as we discuss in the next subsection.

So far, we have observed a vertical spin-separating mechanism for an initial wave packet centered around $\left(x^{\prime}, z^{\prime}\right)=$ $(0.0,4.0)$, where the $z$ inhomogeneity is far greater than the $x$ inhomogeneity. Now, spin density plots are generated for an initial wave packet centered around $\left(x^{\prime}, z^{\prime}\right)=(4.0,4.0)$, where the inhomogeneities in both dimensions are equal (for an initial coherent state in Fig. 9 and an initial incoherent state in Fig. 10). One notices RSS for $|\uparrow\rangle$ and $|\downarrow\rangle$. RSS occurs when the spin components separate along the radial axis. This is understandable since the eigenenergy of the Hamiltonian $H=\frac{p_{x}^{2}+p_{z}^{2}}{2 m}+\mu B_{1}\left(x \sigma_{x}-z \sigma_{z}\right)$ corresponds to a radial linear potential, namely, $\pm \mu B_{1} \sqrt{x^{2}+z^{2}}$. Besides the spin-separation mechanism, one also observes a focusing effect of the component moving toward the transition axis. This effect can be attributed to the difference between the unidirectional linear potential and the radial linear potential. The focusing effect is also consistent with the result in [26]. One also notices that the amplitude for $|\uparrow\rangle$ and $|\downarrow\rangle$ are very different between the $\mathrm{C}$-state case and the IC-state case.

\section{Asymmetry-induced contamination (AIC)}

Now we investigate the dynamics of an initial spin state $|\uparrow\rangle$. Spin density contour plots $\left\langle S_{z}\right\rangle$ are generated for three different cases. All three cases start from an initial location close to the transition axis, the $x$ axis. One observes in Fig. 11 that most spin-ups go up while there is fringe formation between ups and downs close to the $x$ axis which leads to the asymmetry-induced contamination. AIC is observed when the spin component opposite to that predicted by the ideal Stern-Gerlach effect is present. This only occurs when one starts from an asymmetric configuration. In Fig. 11(a), one observes fringes between spin-up and spin-down close to the $x$ axis. In Fig. 11(b) there are fewer fringes when one increases the interaction strength $\mu B_{1}$. Also the spin-up state moves upward more rapidly. Figure 11(c) shows that the wave packet spreads faster in the $z$ direction when the width $w_{z}$ is reduced, and one observes more fringes in both $x$ and $z$ directions.

In order to visualize the AIC, spin density plots are created for $|\uparrow\rangle$ and $|\downarrow\rangle$ separately in Fig. 12. The spin-down state flipped from the spin-up state leads to fringe formation (the butterfly-like pattern in Fig. 12). Nevertheless, the spin-down state amplitude is much smaller than the spin-up. Therefore, the spin-down is only visible in regions where no spin-up is present.

The field in Fig. 2(b) corresponds to a Hamiltonian $H=$ $\frac{p_{x}^{2}+p_{z}^{2}}{2 m}+\mu B_{1}\left(x \sigma_{x}-z \sigma_{z}\right)$. The inclusion of two Pauli matrices complicates the Heisenberg equations of motion. This leads to difficulties in obtaining analytic solutions. In order to obtain a better understanding of the dynamics, we set out to consider the motion in a frame moving with the particle. In such a frame, the system Hamiltonian is $H=\mu B_{1}\left(x \sigma_{x}-z \sigma_{z}\right)$. The corresponding Heisenberg equations of motions are

$$
\begin{gathered}
\dot{\hat{p}}_{x}=-\hat{\sigma}_{x} \mu B_{1}, \quad \dot{\hat{p}}_{z}=\hat{\sigma}_{z} \mu B_{1}, \quad \dot{\hat{x}}=0, \quad \dot{\hat{z}}=0, \\
\dot{\hat{\sigma}}_{x}=\frac{2 \mu B_{1} \hat{z} \hat{\sigma}_{y}}{\hbar}, \quad \dot{\hat{\sigma}}_{z}=\frac{2 \mu B_{1} \hat{x} \hat{\sigma}_{y}}{\hbar}, \\
\dot{\hat{\sigma}}_{y}=-\frac{2 \mu B_{1}\left(\hat{x} \hat{\sigma}_{z}+\hat{z} \hat{\sigma}_{x}\right)}{\hbar} .
\end{gathered}
$$


(a)

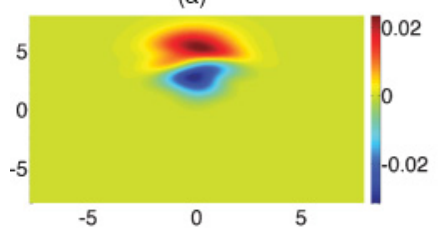

(b)

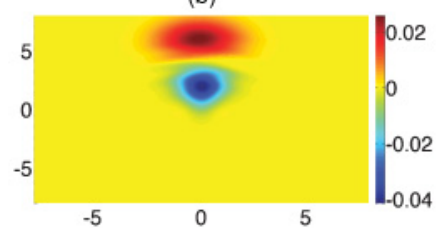

(c)

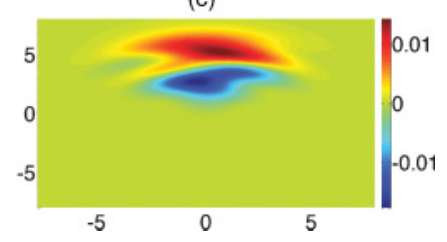

FIG. 7. (Color online) Plots of spin density $\left\langle S_{z}\right\rangle$ at $t=1.0$ (in units of $m d^{2} / \hbar$ ) for an initial C-state $|\uparrow\rangle_{x}$ centered around the $\left(x^{\prime}, z^{\prime}\right)=(0.0,4.0)$ with different sets of interaction strength $\mu B_{1}$ (in units of $\hbar^{2} / m d^{3}$ ) and wave packet widths $w_{x}$ and $w_{z}$ (in units of $d$ ). (a) $\mu B_{1}=2.0, w_{x}=1.0, w_{z}=1.0$, (b) $\mu B_{1}=4.0, w_{x}=1.0, w_{z}=1.0$, (c) $\mu B_{1}=2.0, w_{x}=0.5, w_{z}=1.0$.

(a)

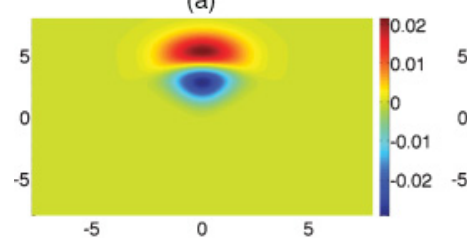

(b)

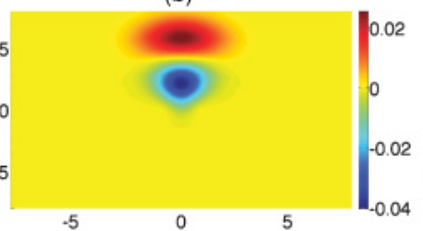

(c)

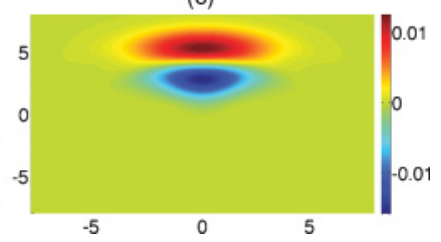

FIG. 8. (Color online) Plots of spin density $\left\langle S_{z}\right\rangle$ at $t=1.0$ (in units of $m d^{2} / \hbar$ ) for a mixed spin state $(50 \%|\uparrow\rangle$ and $50 \%|\downarrow\rangle)$ centered around $\left(x_{0}, z_{0}\right)=(0.0,4.0)$ with different sets of interaction strength $\mu B_{1}$ (in units of $\left.\hbar^{2} / m d^{3}\right)$ and wave packet widths $w_{x}$ and $w_{z}$ (in units of $d$ ). (a) $\mu B_{1}=2.0, w_{x}=1.0, w_{z}=1.0$, (b) $\mu B_{1}=4.0, w_{x}=1.0, w_{z}=1.0$, (c) $\mu B_{1}=2.0, w_{x}=0.5, w_{z}=1.0$.

(a)

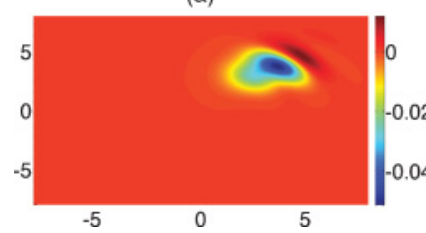

(b)

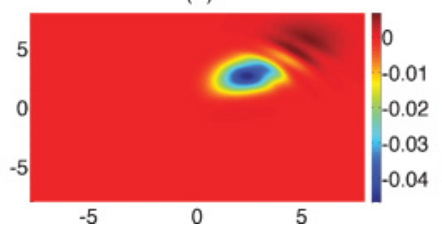

(c)

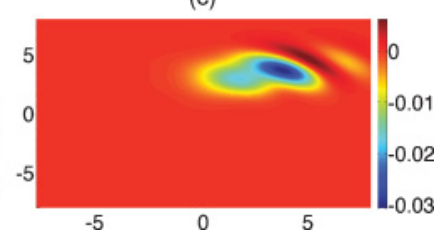

FIG. 9. (Color online) Plots of spin density $\left\langle S_{z}\right\rangle$ at $t=1.0$ (in units of $m d^{2} / \hbar$ ) for an initial C-state $|\uparrow\rangle_{x}$ centered around $\left(x^{\prime}, z^{\prime}\right)=(4.0,4.0)$ with different sets of interaction strength $\mu B_{1}$ (in units of $\hbar^{2} / m d^{3}$ ) and wave packet widths $w_{x}$ and $w_{z}$ (in units of $d$ ). (a) $\mu B_{1}=2.0, w_{x}=$ $1.0, w_{z}=1.0$, (b) $\mu B_{1}=4.0, w_{x}=1.0, w_{z}=1.0$, (c) $\mu B_{1}=2.0, w_{x}=0.5, w_{z}=1.0$.

(a)

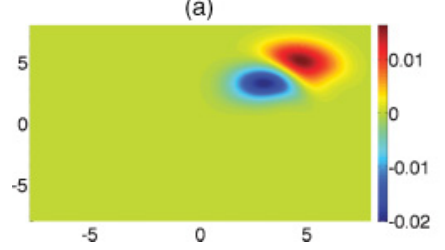

(b)

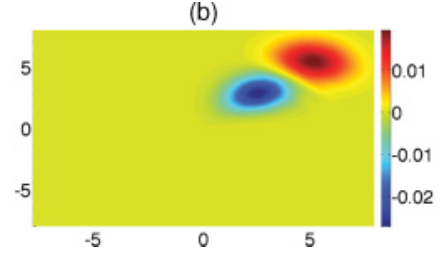

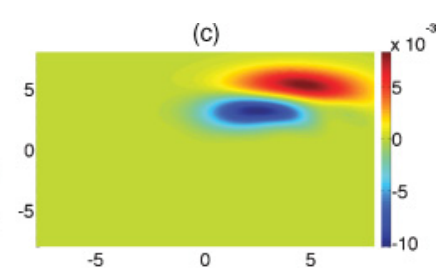

FIG. 10. (Color online) Plots of spin density $\left\langle S_{z}\right\rangle$ at $t=1.0$ (in units of $m d^{2} / \hbar$ ) for an initial IC-state $(50 \%|\uparrow\rangle$ and $50 \%|\downarrow\rangle)$ centered around $\left(x^{\prime}, z^{\prime}\right)=(4.0,4.0)$ with different sets of interaction strength $\mu B_{1}$ (in units of $\hbar^{2} / m d^{3}$ ) and wave packet widths $w_{x}$ and $w_{z}$ (in units of $d$ ). (a) $\mu B_{1}=2.0, w_{x}=1.0, w_{z}=1.0$, (b) $\mu B_{1}=4.0, w_{x}=1.0, w_{z}=1.0$, (c) $\mu B_{1}=2.0, w_{x}=0.5, w_{z}=1.0$.
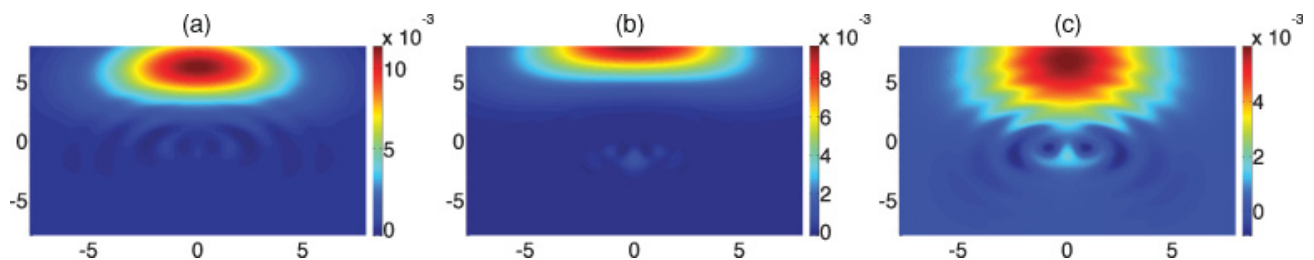

FIG. 11. (Color online) Contour plots of spin density $\left\langle S_{z}\right\rangle$ for an initial spin state $|\uparrow\rangle$ centered around $\left(x^{\prime}, z^{\prime}\right)=(0.0,2.0)$ evaluated at $t=2.0$ (in units of $m d^{2} / \hbar$ ) for different sets of interaction strength $\mu B_{1}$ (in units of $\hbar^{2} / m d^{3}$ ) and wave packet widths $w_{x}$ and $w_{z}$ (in units of $d$ ). (a) $\mu B_{1}=2.0, w_{x}=1.0, w_{z}=1.0$, (b) $\mu B_{1}=3.0, w_{1}=1.0, w_{z}=1.0$, (c) $\mu B_{1}=2.0, w_{x}=1.0, w_{z}=0.5$. 

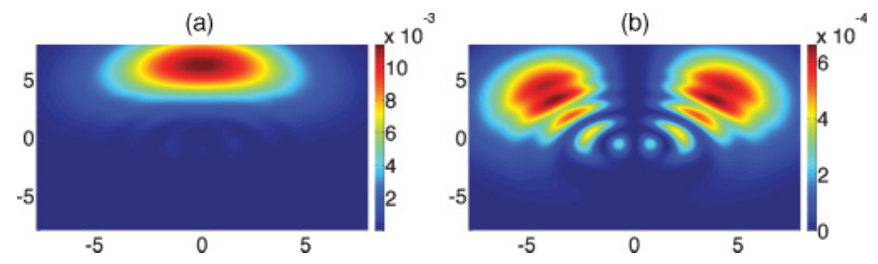

FIG. 12. (Color online) Contour plots of spin densities for an initial spin state $|\uparrow\rangle$ centered around $\left(x^{\prime}, z^{\prime}\right)=(0.0,2.0)$ evaluated at $t=2.0$ (in units of $m d^{2} / \hbar$ ) with $\mu B_{1}=2.0$ (in units of $\hbar^{2} / m d^{3}$ ), $w_{x}=w_{z}=1$ (in units of $d$ ). (a) $\langle\uparrow\rangle$, (b) $\langle\downarrow\rangle$.

It is clear that the position operators are constants of the motion, namely, $x(t)=x(0)$ and $z(t)=z(0)$. It follows that it is easier to calculate the spin dynamics in such a frame. If one assumes an initial $|\uparrow\rangle$ spin state $\left\langle\sigma_{z}\right\rangle(0)=1$, the solution for three spin operators are

$$
\begin{gathered}
\left\langle\sigma_{x}\right\rangle(t)=-\frac{2 x z \sin \left(\frac{\mu B 1 t \sqrt{x^{2}+z^{2}}}{\hbar}\right)^{2}}{x^{2}+z^{2}}, \\
\left\langle\sigma_{y}\right\rangle(t)=-\frac{x \sin \left(\frac{2 \mu B 1 t \sqrt{x^{2}+z^{2}}}{\hbar}\right)}{\sqrt{x^{2}+z^{2}}}, \\
\left\langle\sigma_{z}\right\rangle(t)=\frac{z^{2}+x^{2} \cos \left(\frac{2 \mu B 1 t \sqrt{x^{2}+z^{2}}}{\hbar}\right)}{x^{2}+z^{2}} .
\end{gathered}
$$

In Fig. 13, we display the solutions in three different contour plots, respectively.

The time-dependent spin density $\left\langle\sigma_{z}\right\rangle(t)$ is responsible for AIC. Recall that the solution in Eq. (22) is in a frame where the particle is not moving. "Spin-up goes up" is iconic for the ideal Stern-Gerlach effect. All three figures demonstrate that in a local field similar to the Stern-Gerlach field, the spin-up state has a possibility of moving downward if the initial point is closer to the transition axis ( $x$ axis). This is also an example of nonideal behavior in SGE.

\section{Four-lobes structure}

Figure 14 displays a fringe pattern for $\left\langle S_{x}\right\rangle$ evaluated at $t=1.0$ where the initial state is a C-state $|\uparrow\rangle$ centered around the $z$ axis where the eigenspinors of $S_{z}$ are present. One can also check with features on the $z$ axis and compare it with the BSS structure. There exists reflection asymmetry across the $z$ axis for $\left\langle S_{x}\right\rangle$. In Fig. 14(b), we plot $\left\langle S_{x}\right\rangle$ evaluated at $t=1.0$ where the initial state is a IC-state $(50 \%|\uparrow\rangle$ and $50 \% \mid \downarrow)$ centered around the $z$ axis. It is clear that one cannot see fringe formation, which is consistent with the argument that BSS (a)
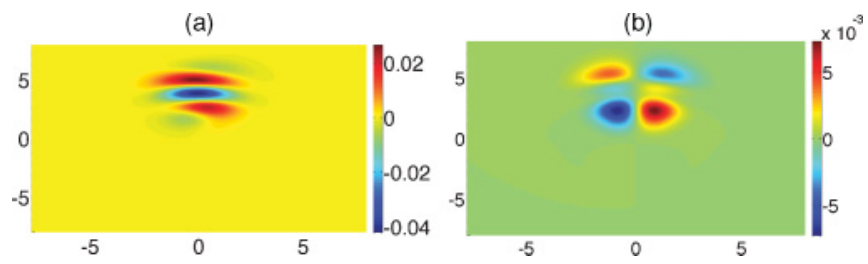

FIG. 14. (Color online) Spin density $\left\langle S_{x}\right\rangle$ for an initial wave packet centered around $\left(x^{\prime}, z^{\prime}\right)=(0.0,4.0)$ (in units of $d$ ) with the following parameter choices: $\mu B_{1}=2.0$ (in units of $\hbar^{2} / m d^{3}$ ), $w_{x}=1.0, w_{z}=1.0$ (in units of d), and $t=1.0$ (in units of $m d^{2} / \hbar$ )

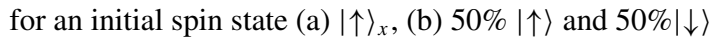

only occurs for an initial C-state. The $|\uparrow\rangle_{x}$ and $|\downarrow\rangle_{x}$ states appear on the right and on the left, and both of them exhibit a beating motion in the vertical direction, namely, oscillatory motion around one point. Note also this motion is also observed for spin-flipping from $|\uparrow\rangle$ to $|\downarrow\rangle$ or vice versa. The IC-state currently in use is in the $z$ basis.

In the 1D-inhomogeneity case, the up and the down states go up and down, respectively, in a symmetric way, and the BSS appears while the polarization is fixed at specific locations. In the 2D-inhomogeneity case, the polarization is not fixed at specific locations but makes periodic oscillations. The only difference between C-states and IC-states is the asymmetric motion for both spin-up and spin-down components displayed by a C-state. For an initial IC-state, one observes a beating pattern of two blobs appearing sideways in the 2D-inhomogeneity case. From the simulations, this beating pattern can also be seen when an initial spin-up flipped to spin-down, which does not happen in the 1D-inhomogeneity case. Therefore, the beating pattern of two blobs might be the consequence of possible spin flipping and asymmetry.

Now, Figs. 15(a) and 15(b) refer to the same initial condition as in Fig. 14 with the time extended to $t=2.0$. The FLS shows separation of $\left\langle S_{x}\right\rangle$ in four quadrants with the same sign across the diagonal. One observes the FLS close to the transition axes. The FLS can be understood in the following way. From the results in the $1 \mathrm{D}$ inhomogeneity, the eigenspinors move toward the corresponding inhomogeneity direction. In the 2D-inhomogeneity case, the $|\uparrow\rangle$ is moving upward if one starts from a $|\uparrow\rangle$ centered around the $z$ axis. However, as pointed out in the radial spin separation, the $|\uparrow\rangle$ goes up and also expands in the $x$ direction. This is very different from the 1D-inhomogeneity case where the symmetry is preserved. The consequence of the asymmetry in the shape of $|\uparrow\rangle$ and $|\downarrow\rangle$ leads to a more complex feature than the BSS on the $z$ axis in the 1D-inhomogeneity case. The $|\downarrow\rangle$ is focused while moving toward the transition axis where the eigenspinors of
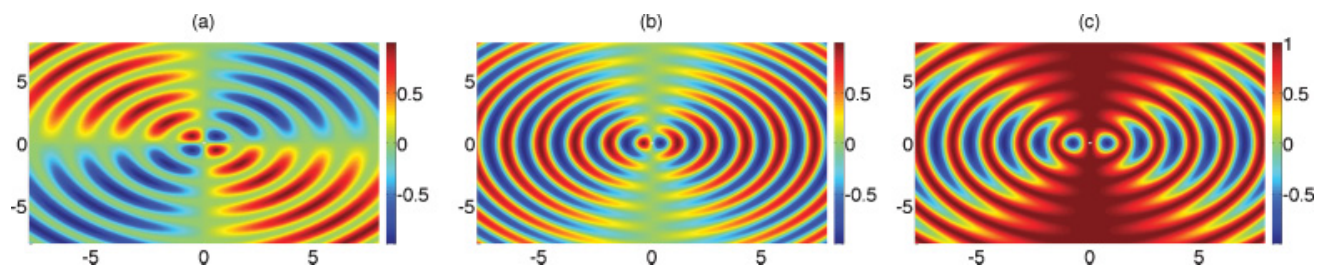

FIG. 13. (Color online) Contour plots of spin densities for an initial spin state $|\uparrow\rangle$ centered around $\left(x^{\prime}, z^{\prime}\right)=(0,0)$ (in units of $\left.d\right)$ in the rest frame with interaction strength $\mu B_{1}=1.0$ (in units of $\hbar^{2} / m d^{3}$ ) and time $t=1.0$ (in units of $m d^{2} / \hbar$ ). (a) $\left\langle\sigma_{x}\right\rangle(t)$, (b) $\left\langle\sigma_{y}\right\rangle(t),\left(\right.$ c) $\left\langle\sigma_{z}\right\rangle(t)$. 


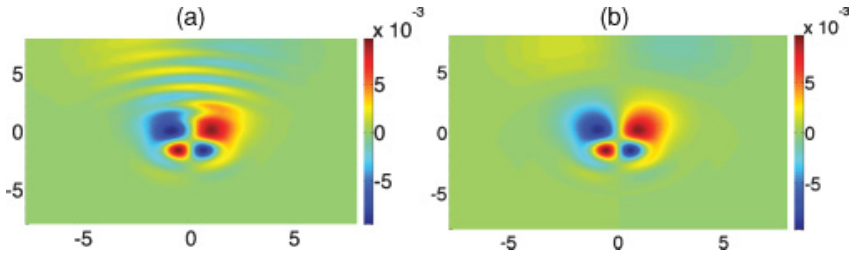

FIG. 15. (Color online) Spin density $\left\langle S_{x}\right\rangle$ for an initial wave packet centered around $\left(x^{\prime}, z^{\prime}\right)=(0.0,4.0)$ (in units of $d$ ) with the following parameter choices: $\mu B_{1}=2.0$ (in units of $\hbar^{2} / m d^{3}$ ), $w_{x}=1.0, w_{z}=1.0$ (in units of $d$ ), and $t=2.0$ (in units of $m d^{2} / \hbar$ )

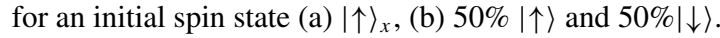

$S_{x}$ are present while $|\uparrow\rangle$ is spreading. The $|\downarrow\rangle$ state can be a superposition of two eigenspinors of $S_{x}$. It should be noted that the $|\uparrow\rangle_{x}$ moves to the left while $|\downarrow\rangle_{x}$ moves to the right as predicted from the inhomogeneity rule. In order to preserve the focusing effect for the $|\downarrow\rangle$, the $|\uparrow\rangle_{x}$ has to be on the right-hand side of $x=0$ and likewise for $|\downarrow\rangle_{x}$. Similarly, for a $|\uparrow\rangle$ centered on the $z$ axis, one expects to see the $|\uparrow\rangle_{x}$ on the left-hand side of $x=0$ and on the right-hand side for $|\downarrow\rangle_{x}$. After moving across the transition axis for $|\downarrow\rangle$, one expects to see the $|\uparrow\rangle_{x}$ and $|\downarrow\rangle_{x}$ states swap sides. This is because after crossing, the $|\downarrow\rangle$ state will expand in size for $z<0$ in the $x$ direction like $|\uparrow\rangle$ for $z>0$. As a result, one can expect a FLS close by the transition axis. This FLS is reminiscent of the nonideal feature in Ref. [26].

\section{DISCUSSION}

We have studied Stern-Gerlach dynamics in both 1D and 2D magnetic-field inhomogeneities using analytic and numerical propagators, respectively. This propagator method enables us to track spin dynamics in time, as shown in spin density $\left\langle S_{i}\right\rangle$ contour plots. We have constructed the analytic propagators for 1D inhomogeneity and have obtained analytic forms for evolved wave packets after applying the integral formula to Gaussian wave packets. In the case where 2D inhomogeneity is present, a difficulty arises with respect to the construction and the application of propagators. The noncommutativity between two Pauli matrices results in the challenge of obtaining an exact solution for the propagators. There simply does not exist a transformation that can decouple the two inhomogeneity directions. Attempting to use the analytic propagators within a short time approximation is inconvenienced by the fact that additional numerical integrations have to be performed to obtain the wave function. Instead, we use a numerical propagation method to propagate the wave packets. This method relies on the use of the Trotter product formula and successive multiplications and Fourier transformations on the wave packet without any space integration.

We have recovered the textbook Stern-Gerlach effect for specific parameter choices. For example, spin separation can be obtained in the following two cases: (1) the beam enters anywhere in an unphysical (violating Maxwell's law) magnetic field with either an initial coherent spin state or an initial spin mixture, and (2) the beam enters the magnetic field (a)

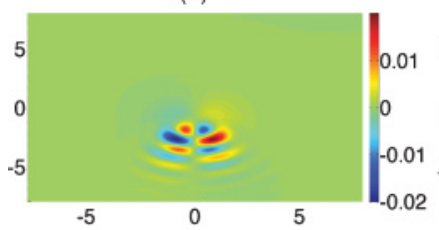

(b)

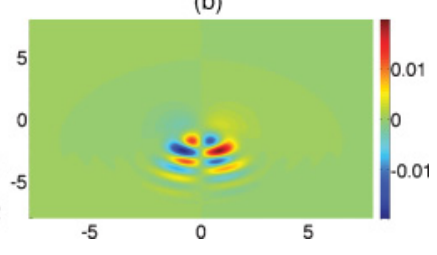

FIG. 16. (Color online) Spin density $\left\langle S_{x}\right\rangle$ for an initial wave packet centered around $\left(x^{\prime}, z^{\prime}\right)=(0.0,4.0)$ (in units of $d$ ) with the following parameter choices: $\mu B_{1}=2.0$ (in units of $\hbar^{2} / m d^{3}$ ), $w_{x}=1.0, w_{z}=1.0$ (in units of $d$ ), and $t=3.0$ (in units of $m d^{2} / \hbar$ ) for an initial spin state (a) $|\uparrow\rangle_{x}$, (b) $50 \%|\uparrow\rangle$ and $50 \%|\downarrow\rangle$.

(2D inhomogeneity with large local magnetic field in $\hat{z}$ ) as long as it enters far above the $x$ axis and close to the $z$ axis. One just has to measure the outcome before the wave packet gets too close to the transition axis. In the first case, the field has global eigenspinors and eigenenergies. As a result, symmetry is preserved and one recovers the vertical Stern-Gerlach separation. In case (2), the presence of two Pauli matrices couples the two dimensions and the field gives position-dependent eigenspinors and eigenenergies. As a result, to get a vertical Stern-Gerlach separation requires a careful choice of initial locations. Indeed, we have shown that a radial separation is obtained when one chooses an arbitrary initial location. Therefore we conclude that a vertical separation is not an automatic feature in a Stern-Gerlach configuration oriented along the $z$ axis. The occurrence of such nonideal SGE features may have interesting ramifications [11].

We compare the wave packet evolution of an initial spin state in either a coherent-state (C-state) representation $\left(|\uparrow\rangle_{x}\right)$

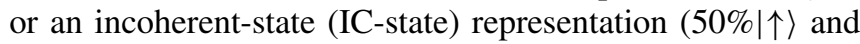
$50 \%|\downarrow\rangle)$. Initial coherent states are used in the few quantum treatments of SGE in the literature. However, this does not reflect the historical Stern-Gerlach experiment where a beam of unpolarized silver atoms is produced out of the oven. The difference between the initial C-state and IC-state leads to different features in both $1 \mathrm{D}$ and 2D cases. In 1D inhomogeneity, we have shown that both the C-state and IC-state exhibit the Stern-Gerlach effect. Nonetheless, the BSS and PSH features only appear with an initial C-state. One sees that the spin polarization is correlated with position and can be controlled by manipulating the interaction strength and wavepacket width of the packet. This makes it a good candidate for a spintronics device. In 2D inhomogeneity, an initial C-state leads to beating patterns.

In conclusion, the propagator method is a powerful tool to study the intricate dynamics of spin-dependent deflection in inhomogeneous fields. It provides us with a representation in real time of spin densities, from which spin currents can be derived. By applying this method to the SGE case, one can not only recover standard Stern-Gerlach spin separation but also visualize nonideal behavior in regions where the two dimensionality of the inhomogeneity matters. The method also allows us to explore the dependence of the effect on the initial coherence of the beam. The features illustrated in this contribution do not exhaust the richness of nonideal Stern-Gerlach dynamics. 
[1] W. Gerlach and O. Stern, Z. Phys. 8, 110 (1922).

[2] D. Herschbach, Ann. Phys. (Leipzig) 10, 163 (2001).

[3] A. Sommerfeld, Ann. Phys. (Leipzig) 51, 17 (1916); 51, 1-94 (1916); 51, 125-167 (1916).

[4] T. E. Phipps and J. B. Taylor, Phys. Rev. 29, 309 (1927).

[5] A. Einstein amd P. Ehrenfest, Z. Phys. 11, 31 (1922).

[6] A. Peres, Quantum Theory: Concepts and Methods (Kluwer, Dordrecht, 1993).

[7] J. J. Sakurai, Modern Quantum Mechanics (Benjamin/ Cummings, Menlo Park, 1985).

[8] J. S. Townsend, A Modern Approach to Quantum Mechanics (University Science Books, Sausalito, CA, 2000).

[9] G. Zhu and C. Singh, PER Conf. Ser. 1179, 309 (2009).

[10] J. Diaz Bulnes and I. S. Oliveira, Braz. J. Phys. 31(3), 488 (2001).

[11] D. Home et al., J. Phys. A 40, 13975 (2007).

[12] A. Sommerfeld, Atombau und Spektrallinien (Friedrich Vieweg \& Sohn, Wiesbaden, 1924).

[13] G. Baym, Lectures on Quantum Mechanics (Benjamin, New York, 1969).

[14] H. Haken and H. C. Wolf, The Physics of Atoms and Quanta: Introduction to Experiments and Theory, 6th ed. (Springer, New York, 2000).

[15] D. Bohm, Quantum Theory (Prentice-Hall, Englewood Cliffs, NJ, 1951).

[16] D. Griffith, Introduction to Quantum Mechanics, 2nd ed. (Pearson Prentice Hall, NJ, 2005).

[17] L. E. Ballentine, Quantum Mechanics: A Modern Development, 1st ed. (World Scientific, Singapore, 1998).

[18] P. Alstrom, Am. J. Phys. 52(3), 275 (1984).

[19] C. Dewdney, P. R. Holland, and A. Kyprianidis, Phys. Lett. A 119(6), 259 (1986).

[20] A. Challinor et al., Phys. Lett. A 218, 128 (1996).

[21] E. B. Manukian, Quantum Theory: A Wide Spectrum, 1st ed. (Springer, New York, 2006).
[22] G. A. Gallup, H. Batelaan, and T. J. Gay, Phys. Rev. Lett. 86, 4508 (2001).

[23] E. Kennard, Z. Phys. 44, 326 (1927).

[24] M. O. Scully, R. Shea, and J. D. McCullen, Phys. Rep. 43, 485 (1978).

[25] M. O. Scully, W. E. Lamb Jr., and A. O. Barut, Found. Phys. 17, 575 (1987).

[26] G. Potel, F. Barranco, S. Cruz-Barrios, and J. Gomez-Camacho, Phys. Rev. A 71, 052106 (2005).

[27] B. C. Hsu and J.-F. S. Van Huele, Phys. Rev. B 80, 235309 (2009).

[28] Eugen Merzbacher, Quantum Mechanics, 3rd ed. (Wiley, New York, 1998).

[29] Q. Wang, J. Phys. A 20, 5041 (1987).

[30] F. A. Barone et al., Am. J. Phys. 71, 483 (2003).

[31] G. Y. Tsaur and J. Wang, Am. J. Phys. 74, 600 (2006).

[32] L. S. Brown and Y. Zhang, Am. J. Phys. 62, 806 (1994).

[33] G. P. Arrighini, N. L. Durante, and C. Guidotti, Am. J. Phys. 64, 1036 (1996).

[34] R. P. Feynman, Quantum Mechanics and Path Integrals (McGraw-Hill, New York, 1965).

[35] E. I. Rashba, Fiz. Tverd. Tela (Leningrad) 2, 1224 (1960); Sov. Phys. Solid State 2, 1109 (1960).

[36] G. Dresselhaus, Phys. Rev. 100, 580 (1955).

[37] R. Winkler, Spin-Orbit Coupling Effects in Two-Dimensional Electron and Hole Systems (Springer, Berlin, 2003).

[38] H. De Raedt and B. De Raedt, Phys. Rev. A 28, 3575 (1983).

[39] J. Katriel, J. Phys. A: Math. Theor. 16, 4171 (1983).

[40] More precisely, the point is a saddle point of the magnetic scalar potential $\Phi_{M}=\frac{B_{1}}{2}\left(x^{2}-z^{2}\right)$, from which the field can be derived $B=-\nabla \Phi_{M}=\left(-B_{1} x, 0, B_{1} z\right)$.

[41] D. Kosloff and R. Kosloff, J. Comput. Phys. 52, 35 (1983). 